\title{
Advancements in Gesture Recognition Technology
}

\author{
${ }^{1}$ Poluka Srilatha, ${ }^{2}$ Tiruveedhula Saranya \\ ${ }^{1,2}$ Department of Electronics and Communication Engineering,Sree Vidyanikethan Engineering College \\ (Autonomous),(Affiliated to JNTU, Anantapur), Sree Sainath Nagar, Tirupathi, India.
}

\begin{abstract}
Gesture recognition is an upcoming technology which can change entire technology and our work in daily life. Gesture means signs made by human beings which originate from face, hands or any part of the body. These gestures can be captured using scanning or video methods and by processing these gestures in to human signals.

GUI related interfaces are used in which text is taken as input from mouse and keyboard. In this new system gestures are used as inputs which do not require any mechanical elements to communicate between man and machine. If we move our hand on computer screen and based on our movement, curser will accordingly move which will make work easier. But now with increase in technological knowledge, the concept slowly enhanced into voice, speech recognition and position recognition models. These have enriched the domain and have roped in some very sophisticated means of human computer interaction. Finger tracking is one such advanced gesture innovation. It is the use of hands and their various positions to kick-start a computer application. It aims at minimizing the use of keyboard and mouse. Non-touch based interaction or giving the input to computers with eyes is one major breakthrough in the domain. It can certainly be adjudged as the ray of hope for disabled people or people busy with multitasking.
\end{abstract}

Keywords: Algorithm, AllSee, GUI, HMI, sign language.

\section{Introduction}

Gesture recognition can be seen as a way for computers to begin to understand human body language, thus building a richer bridge between machines and humans than primitive interfaces or even GUIs (graphical user interfaces), which still limit the majority of input to keyboard and mouse.

Gesture recognition enables humans to communicate with the machine (HMI) and interact naturally without any mechanical devices. Using the concept of gesture recognition, it is possible to point a finger at the computer screen so that the cursor will move accordingly. This could potentially make conventional input devices such as mouse, keyboards_and even touch-screens_redundant. Gesture recognition can be conducted with techniques from computer vision and image processing. The literature includes ongoing work in the computer vision field on capturing gestures or more general human pose and movements by cameras connected to a computer.

1 Types of gestures

\section{Headings}

1.1 online gestures

1.2 offline gestures

2 Various types of gestures recognised by computer

2.1 Sign language recognition

2.2 For socially assistive robotics

2.3 Control through facial gestures

2.4 Alternative computer interfaces

2.5 Immersive game technology

2.6 Remote control

3 Brief history of gesture recognition technology

4 Input devices

4.1 Wired gloves

4.2 Depth aware camera

4.3 Stereo camera

4.4 Controller based gestures

4.5 Single camera

5 A basic working of the gesture recognition system

6 Challenges 


\section{Conclusion}

Figure 2.3

Figure 2.4

Figure 2.5

Figure 2.6

Figure 3.1

Figure 3.2

Figure 3.3

Figure 3.4

Figure 3.5

Figure 3.6

Figure 4.1

Figure 4.2

Figure 4.4

Figure 5.1

Figure 5.2

\section{Figures}

Controlling computer through facial gestures

Alternate computer interface

Gestures to control interactions within video games

Remote control with the wave of hand

First touch screen device

First personal computer

The essential reality p5 glove

Apple iphone

Multi-touch product by Microsoft

Application of open frame works

Wired gloves

Depth aware camera

Controller based gestures

working of gesture recognition system

Types of algorithms

\section{Types of gestures}

In computer interfaces, two types of gestures are distinguished. We consider online gestures, which can also be regarded as direct manipulations like scaling and rotating. In contrast, offline gestures are usually processed after the interaction is finished; e. g. a circle is drawn to activate a context menu.

\subsection{Online gestures:}

Direct manipulation gestures. They are used to scale or rotate a tangible object.

1.2 Offline gestures:

Those gestures that are processed after the user interaction with the object. An example is the gesture to activate a menu.

\section{Various types of gestures recognised by computer}

Gesture recognition is useful for processing information from humans that is not conveyed through speech or type. There are also various types of gestures that can be identified by computers.

\subsection{Sign language recognition:}

Just as speech recognition can transcribe speech to text, certain types of gesture recognition software can transcribe the symbols represented through sign language into text.

\subsection{For socially assistive robotics:}

By using proper sensors (accelerometers and gyros) worn on the body of a patient and by reading the values from those sensors, robots can assist in patient rehabilitation. The best example can be stroke rehabilitation.

\subsection{Control through facial gestures:}

Controlling a computer through facial gestures is a useful application of gesture recognition for users who may not physically be able to use a mouse or keyboard. Eye tracking in particular may be of use for controlling cursor motion or focusing on elements of a display.

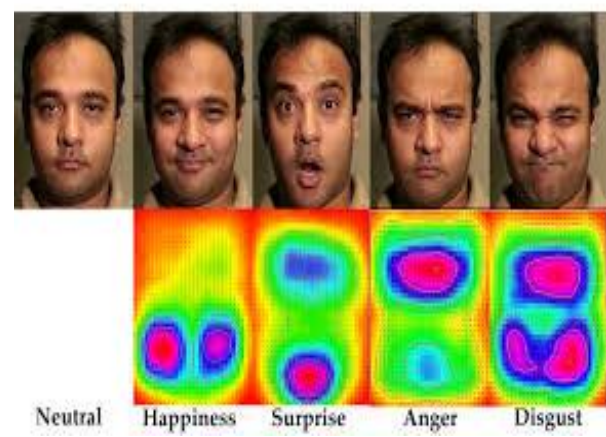

Fig 2.3: Controlling computer through facial gestures. 


\subsection{Alternative computer interfaces:}

Fore going the traditional keyboard and mouse setup to interact with a computer, strong gesture recognition could allow users to accomplish frequent or common tasks using hand or face gestures to a camera.

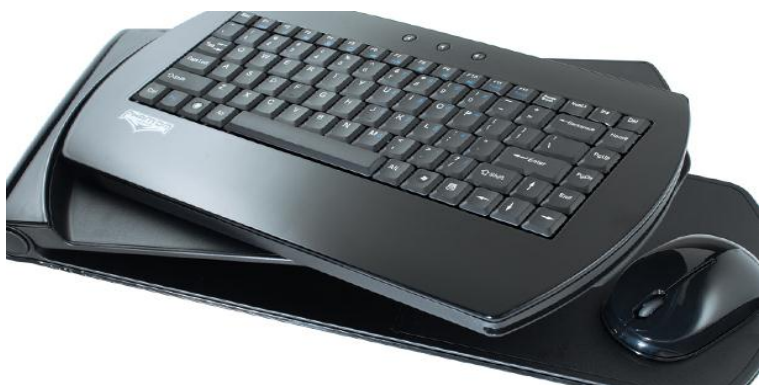

Fig 2.4: Alternate computer interface.

\subsection{Immersive game technology:}

Gestures can be used to control interactions within video games to make the game player's experience more interactive or immersive.

\subsection{Remote control:}

Fig 2.5: Gestures to control interactions within video games.

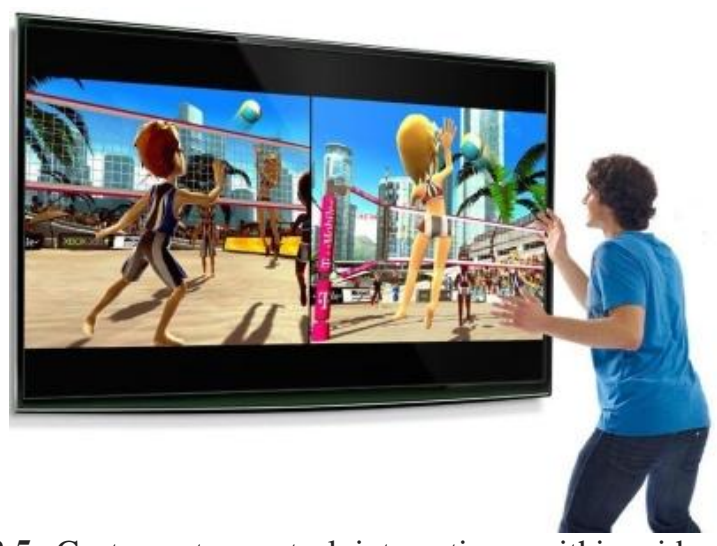

Through the use of gesture recognition, remote control with the "wave of a hand" of various devices is possible. The signal must not only indicate the desired response, but also which device to be controlled.

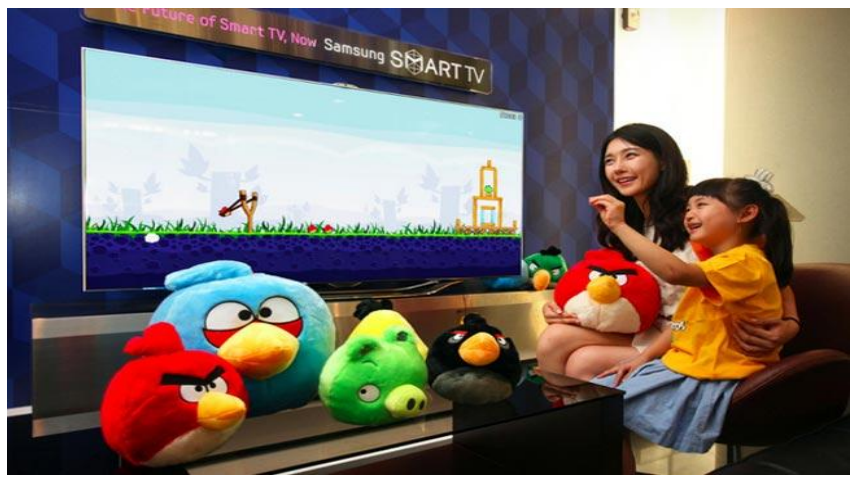

Fig 2.6: Remote control with the wave of hand.

\section{7: Accutouch}

\section{Brief History Of Gesture Recognition Technology}

The first true touch screen device in the form of a curved glass touch screen sensor. 


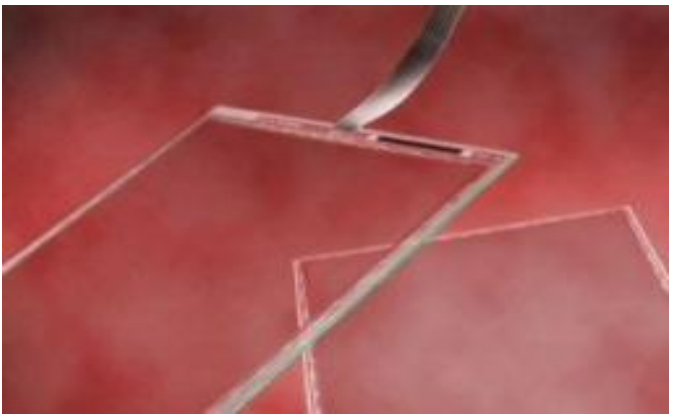

Fig 3.1: First touch screen device.

\section{3: Hewlett-packard 150}

The first personal computer featuring a touch-sensitive screen allows users to position the cursor and select on-screen buttons.

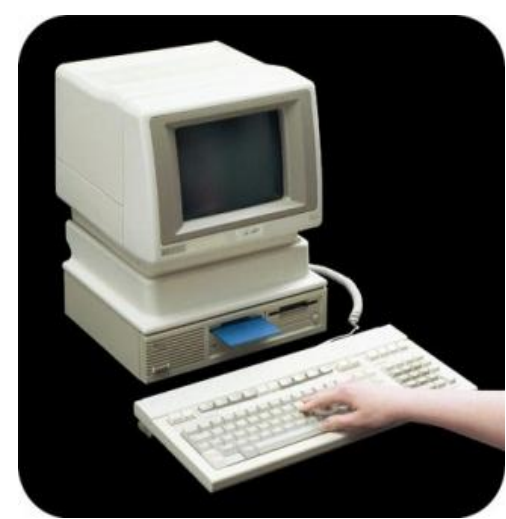

Fig 3.2: First personal computer.

2001: The Essential Reality P5 Glove By Lionhead Studios

Black \& White is a game controlled by a special glove that can translate physical gestures into movement on the screen. This is likely the first commercial controller for gestural interfaces.

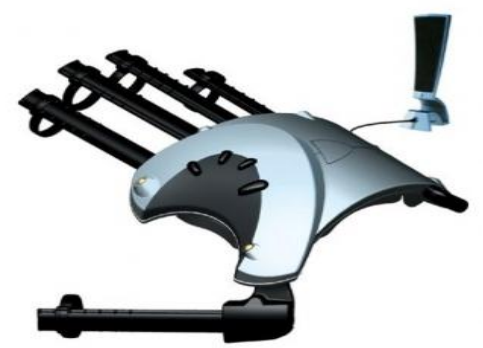

Fig 3.3: the essential reality 5 glove

2007:Apple announces the iphone

Apple receives a patent for an Apple touch device using gestural interface.
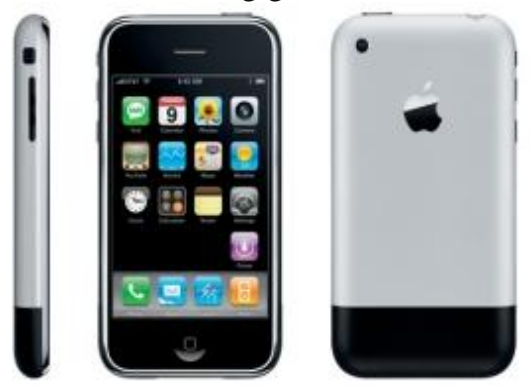

Fig 3.4: Apple iphone. 


\section{Microsoft surface}

Microsoft announces a multi-touch product that combines software and hardware to offer image manipulation through hand gestures and physical objects.

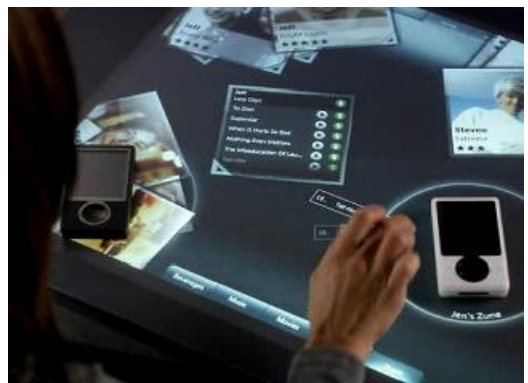

Fig 3.5: Multi-touch product by Microsoft.

\section{8/9: Open frameworks}

New programming platforms such as Open Frameworks create simple tools for developing highly interactive interfaces that can be easily and intuitively triggered and manipulated. A fun example of an Open Frameworks application is Sniff, an interactive storefront window display of an animated dog that follows passers-by, discerns their behaviour and engaging them in play.

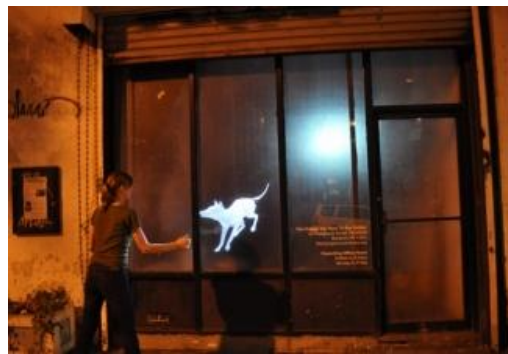

Figure 3.6: Application of open frame works.

\section{Input devices}

The ability to track a person's movements and determine what gestures they may be performing can be achieved through various tools. Although there is a large amount of research done in image/video based gesture recognition, there is some variation within the tools and environments used between implementations.

\subsection{Wired gloves:}

These can provide input to the computer about the position and rotation of the hands using magnetic or inertial tracking devices. Furthermore, some gloves can detect finger bending with a high degree of accuracy or even provide haptic feedback to the user, which is a simulation of the sense of touch. The first commercially available hand-tracking glove-type device was the Data Glove, a glove-type device which could detect hand position, movement and finger bending.

\subsection{Depth-aware camera:}

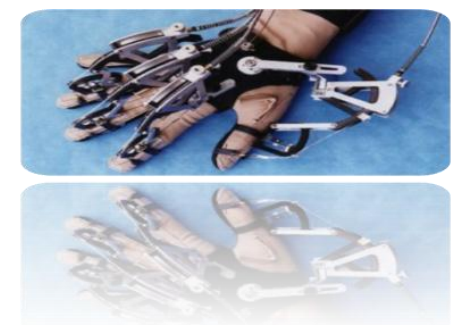

Fig 4.1: Wired gloves.

Using specialized cameras such as structured light or time-of-flight cameras, one can generate a depth map of what is being seen through the camera at a short range, and use this data to approximate a $3 \mathrm{~d}$ representation of what is being seen. These can be effective for detection of hand gestures due to their short range capabilities. 


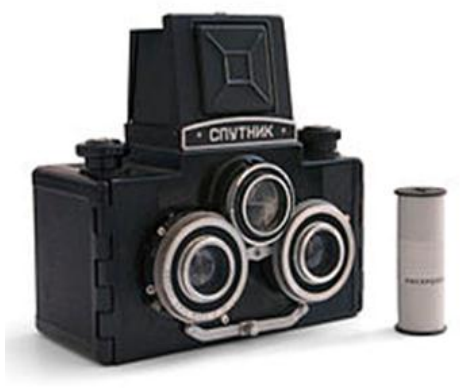

Fig 4.2: Depth aware camera.

\subsection{Stereo camera:}

Using two cameras whose relations to one another are known, a $3 \mathrm{~d}$ representation can be approximated by the output of the cameras. In combination with direct motion measurement (6D-Vision) gestures can directly be detected.

\subsection{Controller-based gestures:}

These controllers act as an extension of the body so that when gestures are performed, some of their motion can be conveniently captured by software. Mouse gestures are one such example, where the motion of the mouse is correlated to symbol being drawn by a person's hand.

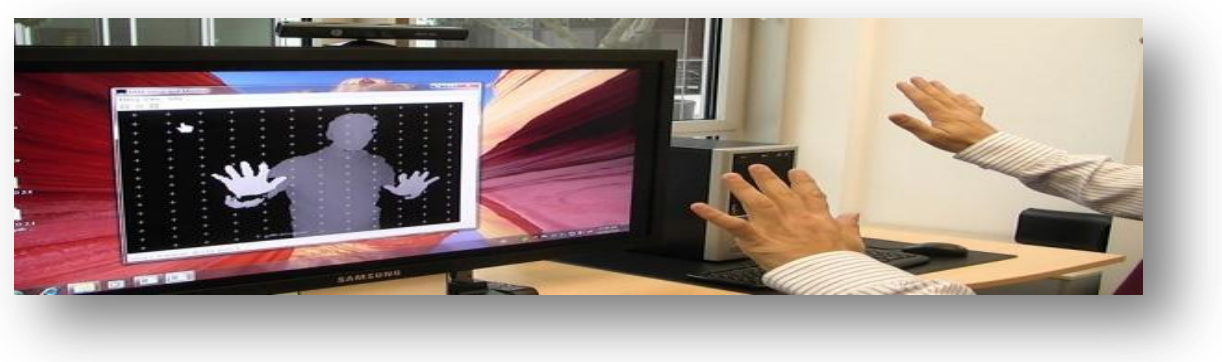

Fig 4.4: Controller based gestures.

\subsection{Single camera:}

A standard 2D camera can be used for gesture recognition where the resources/environment would not be convenient for other forms of image-based recognition. Earlier it was thought that single camera may not be as effective as stereo or depth aware cameras, but some companies are challenging this theory.

\section{A Basic Working Of The Gesture Recognition System}

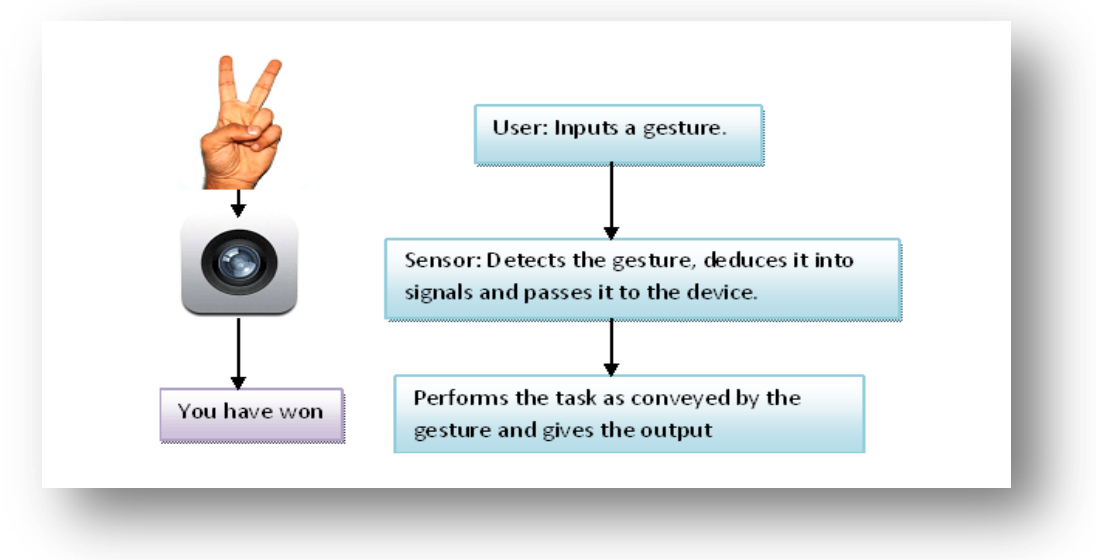

Fig 5.1: working of gesture recognition system. 


\section{Algorithm:}

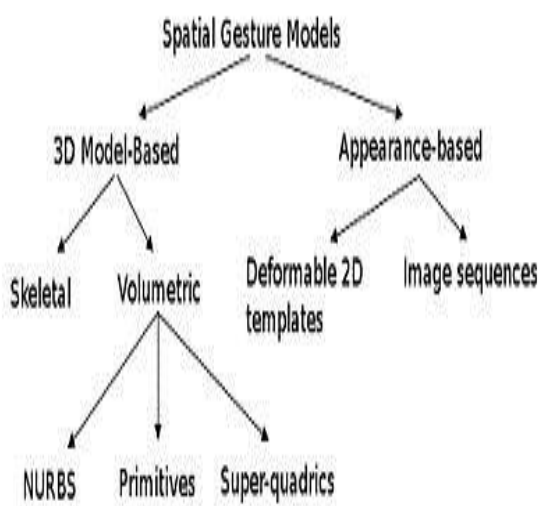

Fig 5.2: Types of algorithms.

\section{Challenges}

There are many challenges associated with the accuracy and usefulness of gesture recognition software. For image-based gesture recognition there are limitations on the equipment used and image noise. Images or video may not be under consistent lighting, or in the same location. Items in the background or distinct features of the users may make recognition more difficult.

The variety of implementations for image-based gesture recognition may also cause issue for viability of the technology to general usage.

\section{Conclusion}

Gesture recognition technology is relatively robust and accurate. Trade off can be maintained between speed and accuracy. Non-touch based interaction can certainly be adjudged as the ray of hope for disabled people or people busy with multitasking.

\section{References}

[1]. http://en.wikipedia.org/wiki/Facial_recognition_system

[2]. http://en.wikipedia.org/wiki/Gesture_recognition

[3]. https://goldin-meadow-lab.uchicago.edu/sites/goldin-meadow lab.uchicago.edu/files/uploads/PDFs/1999_GM.pdf 